(2) Open Access Full Text Article

\title{
Acquisition of T790M resistance mutation in a patient with advanced adenocarcinoma harboring uncommon EGFR mutations: a case report and literature review
}

This article was published in the following Dove Medical Press journal: OncoTargets and Therapy

\section{Taiki Hakozaki \\ Makiko Yomota \\ Department of Thoracic Oncology and Respiratory Medicine, Tokyo Metropolitan Cancer and Infectious Diseases Center Komagome Hospital, Tokyo, Japan}

Background: Osimertinib is the standard of care for patients with non-small-cell lung cancer (NSCLC) patients harboring acquired EGFR T790M resistance mutation. However, the frequency of T790M resistance mutation acquisition and the efficacy of osimertinib in patients harboring uncommon EGFR mutations, which accounts for approximately $10 \%$ of $E G F R$ mutations, remain unclear.

Case presentation: We present the case of a 72-year-old Japanese woman with recurrent NSCLC harboring uncommon EGFR mutations, who was subsequently found to have acquired the T790M resistance mutation and was treated with osimertinib. She died 9 days later because of worsening respiratory failure with disease progression.

Conclusion: The findings of the present case suggest that the T790M resistance mutation can occur even when the patient harbors an uncommon EGFR mutation after EGFR-tyrosine kinase inhibitor treatment, and the prognosis could be poor despite the presence of an acquired T790M resistance mutation.

Keywords: non-small-cell lung cancer, EGFR-TKIs, osimertinib, uncommon EGFR mutation, acquired resistance

\section{Introduction}

$E G F R$ mutations are some of the major mutations in non-small-cell lung cancer (NSCLC). Exon 19 deletion and exon 21 L858R point mutation, which are referred to as common $E G F R$ mutations, are activating EGFR mutations associated with $E G F R$ tyrosine kinase inhibitors (TKIs). Approximately $10 \%$ of EGFR mutations represent uncommon EGFR mutations. ${ }^{1}$ First-generation $E G F R$-TKIs show insufficient clinical benefit for uncommon EGFR mutations; ${ }^{2}$ however, second-generation EGFR-TKIs, such as afatinib, show a $60 \%$ response rate. ${ }^{3}$ Because prospective data on survival are absent and uncommon EGFR mutations are heterogeneous, there is no established standard of care (SOC) for patients harboring uncommon EGFR mutations.

Acquired resistance to EGFR-TKIs develops after a median of 10-12 months, and it is most commonly mediated by an EGFR exon 20 T790M mutation. The AURA 3 Phase III trial demonstrated the superiority of osimertinib over platinum therapy plus pemetrexed, which had been the SOC for patients with NSCLC harboring acquired resistance to prior $E G F R$-TKIs. ${ }^{4}$ However, the frequency of T790M resistance mutation acquisition after treatment with EGFR-TKIs for uncommon EGFR mutations remains 
unclear, although $50 \%-60 \%$ of cases of $E G F R$ common mutations, including exon 19 deletion and exon 21 L858R mutation, acquire the T790M resistance mutation. Under these circumstances, the optimal treatment approach for patients harboring uncommon EGFR mutations remains unclear.

Herein, we present the case of a patient with recurrent NSCLC harboring uncommon EGFR mutations, who was subsequently found to have acquired the T790M resistance mutation and was treated with osimertinib.

\section{Case presentation}

A 72-year-old Japanese woman presented with abnormal chest opacity at an annual health checkup. She was a nonsmoker and had no specific medical history. Her Eastern Cooperative Oncology Group (ECOG) performance status was zero. Chest computed tomography (CT) revealed a pulmonary nodule measuring $2.5 \times 1.6 \mathrm{~cm}$ in the left lower lobe. She underwent left lower lobectomy and systemic lymph node dissection. Based on the analysis of the nodule, she was diagnosed with adenocarcinoma (pT2aN2M0 Stage IIIA) harboring an EGFR exon 18 G719X mutation. She underwent four cycles of adjuvant chemotherapy with cisplatin plus vinorelbine. Recurrence with multiple intrapulmonary metastases and malignant pleural effusion were observed later. Genetic analysis of the pleural effusion at that time showed an EGFR exon 18 G719X mutation, as previously detected. Subsequently, she was treated with gefitinib for 15 months, and then, increased pleural effusion and carcinomatous lymphangiomatosis were noted. Both EGFR exon 18 G719X and exon 20 T790M mutations were detected in her plasma (Cobas ${ }^{\mathrm{TM}}$ EGFR Mutation Test v2, Hoffman-La Roche Ltd., Basel, Switzerland). Her ECOG performance status declined to 2 because of worsening dyspnea, and she was treated with osimertinib. She died 9 days later from worsening respiratory failure with disease progression.

\section{Discussion and conclusion}

Tumor genotyping for an acquired T790M resistance mutation at disease progression has become a standard component of care in patients with NSCLC harboring EGFR mutations to guide subsequent treatment. Previous studies have shown that approximately $50-60 \%$ of patients treated with first or second-generation EGFR-TKIs acquire the T790M resistance mutation at the time of progressive disease (PD). However, in these studies, almost all patients had common EGFR mutations, such as exon 19 deletion and exon 21 L858R mutation. Therefore, the prevalence of T790M resistance mutation acquisition in patients harboring uncommon EGFR mutations and the efficacy of third-generation EGFR-TKIs in this population remain unclear.

In the AURA Phase I/II trial, osimertinib was administered to 222 patients with NSCLC harboring both T790M-mediated and non-T790M-mediated resistance to EGFR-TKIs in the expansion cohort. Of these 222 patients, 10 (4.5\%) initially had uncommon EGFR mutations, and 4 of these 10 patients (40\%) acquired the T790M resistance mutation. ${ }^{5}$ In a study of 125 patients who were re-biopsied at disease progression with initial EGFR-TKIs (mostly first-generation therapy), only $6(5 \%)$ initially had uncommon mutations, and only 1 of these 6 patients $(17 \%)$ acquired the $7790 \mathrm{M}$ resistance mutation. ${ }^{6}$ In another study on 37 patients who were treated with afatinib, $3(8 \%)$ initially had uncommon EGFR mutations, and none of these 3 patients $(0 \%)$ acquired the $T 790 \mathrm{M}$ resistance mutation at the time of PD (Table 1). ${ }^{7}$ These results may indicate that patients with NSCLC harboring uncommon EGFR mutations

Table I Patients with uncommon EGFR mutations who were subsequently treated with osimertinib in previous studies

\begin{tabular}{|c|c|c|c|}
\hline & $\begin{array}{l}\text { EGFR mutation status at the } \\
\text { baseline }\end{array}$ & $\begin{array}{l}\text { T790M } \\
\text { status } \\
\text { at PD }\end{array}$ & $\begin{array}{l}\text { Best } \\
\text { objective } \\
\text { response }\end{array}$ \\
\hline \multicolumn{4}{|c|}{ AURA $(\mathrm{N}=222)$} \\
\hline I & Exon 18 G7I9X & $(+)$ & PR \\
\hline 2 & Exon $18 \mathrm{G} 719 \mathrm{X}$ & $(+)$ & SD \\
\hline 3 & Exon I8 G7I9X & $(+)$ & SD \\
\hline 4 & Exon $18 \mathrm{G} 7 \mathrm{I}$ X+exon $20 \mathrm{~S} 768 \mathrm{I}$ & $(+)$ & PR \\
\hline 5 & Exon 18 G7I9X & $(-)$ & SD \\
\hline 6 & Exon 18 G7I9X & $(-)$ & PD \\
\hline 7 & Exon I8 G7I9X & $(-)$ & PD \\
\hline 8 & Exon $18 \mathrm{G} 719 \mathrm{X}$ & $(-)$ & PD \\
\hline 9 & Exon I8 G7।9X+exon 20 S768I & $(-)$ & SD \\
\hline 10 & Exon 20 insertion & $(-)$ & PD \\
\hline \multicolumn{4}{|c|}{ Kawamura et $\mathrm{al}^{7}(\mathbf{N}=\mathbf{1 2 5})$} \\
\hline I & Uncommon EGFR mutation ${ }^{\mathrm{a}}$ & $(+)$ & NA \\
\hline 2 & Uncommon EGFR mutation ${ }^{\mathrm{a}}$ & $(-)$ & NA \\
\hline 3 & Uncommon EGFR mutation ${ }^{\mathrm{a}}$ & $(-)$ & NA \\
\hline 4 & Uncommon EGFR mutation ${ }^{\mathrm{a}}$ & $(-)$ & NA \\
\hline 5 & Uncommon EGFR mutation ${ }^{\mathrm{a}}$ & $(-)$ & NA \\
\hline 6 & Uncommon EGFR mutation ${ }^{\mathrm{a}}$ & $(-)$ & NA \\
\hline \multicolumn{4}{|c|}{ Tanaka et al $^{6}(\mathbf{N}=37)$} \\
\hline I & Exon $18^{b}$ & $(-)$ & NA \\
\hline 2 & Exon $20^{\mathrm{b}}$ & $(-)$ & NA \\
\hline 3 & Exon $20^{b}$ & $(-)$ & NA \\
\hline
\end{tabular}

Notes: a'Exon 18 G7I9X, exon 20 insertion, or exon 2I L86IQ. ${ }^{\text {DD }}$ etails were not provided.

Abbreviations: PR, partial response; SD, stable disease; PD, progressive disease; NA, not available. 
are less likely to acquire the T790M resistance mutation when compared with those who have common EGFR mutations (50\%-60\%). In the AURA 2 Phase II $(\mathrm{N}=210)$ and AURA 3 Phase III ( $\mathrm{N}=419)$ trials, of all participants who had acquired T790M resistance, 8 of 210 (4\%) and 11 of 419 (3\%) participants initially had uncommon EGFR mutations. ${ }^{4,8}$ Considering the prevalence of uncommon EGFR mutations among all EGFR mutations (approximately $10 \%$ ), the proportion of patients harboring uncommon EGFR mutations in these trials was less. This may reflect the rarity of T790M resistance acquisition among patients harboring uncommon EGFR mutations.

In addition, there are limited data on the efficacy of osimertinib among patients with T790M resistance acquisition who previously had uncommon EGFR mutations besides the 4 patients in the AURA trial. Of these 4 patients, 2 showed partial response $(\mathrm{PR})$ and 2 showed stable disease $(\mathrm{SD})$ as the best objective response (Table 1). In contrast, in the present case, osimertinib was found to be inactive with rapid disease progression. We cannot dismiss the poor performance status of the patient at the time of osimertinib initiation; however, her clinical course may have reflected characteristics of the rare EGFR mutation status. The contribution of mechanisms of acquired resistance other than T790M mutation may have been substantial, and cytotoxic chemotherapy could be a choice of treatment if the general condition permits. Prior studies and the present case describe some characteristics of patients with T790M resistance mutation acquisition who previously had uncommon EGFR mutations. However, further cases should be accumulated to obtain practical clinical information that can guide optimal treatment. In addition, it may be reasonable to utilize comprehensive methods, such as next-generation sequencing, particularly in small patient subgroups for which conformational clinical trials may not be feasible.

The findings of the present case indicate that the T790M resistance mutation can occur even when the patient harbors an uncommon EGFR mutation after EGFR-TKI treatment and that the prognosis could be poor despite the presence of an acquired T790M resistance mutation. Further understanding of the clinical characteristics may help in the optimization of strategies to provide the maximum clinical benefit in patients with NSCLC harboring uncommon EGFR mutations.

\section{Ethics approval and informed consent}

The case report was waivered by the Ethics Committee of Tokyo Metropolitan Cancer and Infectious diseases Center
Komagome Hospital. The clinical information presented in this case report was obtained through Tokyo Metropolitan Cancer and Infectious Diseases Center Komagome Hospital's medical records.

\section{Consent for publication}

Written informed consent was obtained from the patient for publication of this case report.

\section{Data availability}

All relevant data are within the manuscript.

\section{Acknowledgments}

The authors would like to thank Enago (https://www. enago.jp) for the English language review. This report did not receive any specific grant from funding agencies in the public, commercial, or not-for-profit sectors.

\section{Author contributions}

Both authors contributed to data analysis, drafting and revising the article, gave final approval of the version to be published, and agree to be accountable for all aspects of the work.

\section{Disclosure}

The authors report no conflicts of interest in this work.

\section{References}

1. Tu HY, Ke EE, Yang JJ, et al. A comprehensive review of uncommon EGFR mutations in patients with non-small cell lung cancer. Lung Cancer. 2017;114:96-102.

2. Watanabe S, Minegishi Y, Yoshizawa H, et al. Effectiveness of gefitinib against non-small-cell lung cancer with the uncommon EGFR mutations G719X and L861Q. J Thorac Oncol. 2014;9(2):189-194.

3. Yang JC, Sequist LV, Geater SL, et al. Clinical activity of afatinib in patients with advanced non-small-cell lung cancer harbouring uncommon EGFR mutations: a combined post-hoc analysis of LUX-Lung 2, LUX-Lung 3, and LUX-Lung 6. Lancet Oncol. 2015;16(7):830-838.

4. Mok TS, Wu Y-L, Ahn M-J, et al. Osimertinib or platinum-pemetrexed in EGFR T790M-positive lung cancer. N Engl J Med. 2017;376(7):629-640.

5. Jänne PA, Yang JC, Kim DW, et al. AZD9291 in EGFR inhibitor-resistant non-small-cell lung cancer. N Engl J Med. 2015;372(18):1689-1699.

6. Tanaka K, Nosaki K, Otsubo K, et al. Acquisition of the T790M resistance mutation during afatinib treatment in EGFR tyrosine kinase inhibitor-naïve patients with non-small cell lung cancer harboring $E G F R$ mutations. Oncotarget. 2017;8(40):68123-68130.

7. Kawamura T, Kenmotsu H, Omori S, et al. Clinical factors predicting detection of T790M mutation in rebiopsy for EGFR-mutant non-smallcell lung cancer. Clin Lung Cancer. 2018;19(2):e247-e252.

8. Goss G, Tsai CM, Shepherd FA, et al. Osimertinib for pretreated EGFR Thr790Met-positive advanced non-small-cell lung cancer (AURA2): a multicentre, open-label, single-arm, phase 2 study. Lancet Oncol. 2016; 17(12):1643-1652. 


\section{Publish your work in this journal}

OncoTargets and Therapy is an international, peer-reviewed, open access journal focusing on the pathological basis of all cancers, potential targets for therapy and treatment protocols employed to improve the management of cancer patients. The journal also focuses on the impact of management programs and new therapeutic agents and protocols on

patient perspectives such as quality of life, adherence and satisfaction. The manuscript management system is completely online and includes a very quick and fair peer-review system, which is all easy to use. Visit http://www.dovepress.com/testimonials.php to read real quotes from published authors.

Submit your manuscript here: http://www.dovepress.com/oncotargets-and-therapy-journal 